Moroccan J. of Pure and Appl. Anal. (MJPAA)

Volume 6(2), 2020, Pages 155-167

ISSN: Online 2351-8227 - Print 2605-6364

DOI: $10.2478 /$ mjpaa-2020-0012

\title{
A new generalization of two refined Young inequalities and applications
}

\section{A. IghaChane ${ }^{1}$ AND M. AKKouchi ${ }^{2}$}

Авstract. In this paper, we prove that if $a, b>0$ and $0 \leq \alpha \leq 1$, then for $m=1,2,3, \ldots$,

$$
\begin{aligned}
r_{0}^{m}\left(a^{\frac{m}{2}}-b^{\frac{m}{2}}\right)^{2} & \leq r_{0}^{m}\left(\frac{b^{m+1}-a^{m+1}}{b-a}-(m+1)(a b)^{\frac{m}{2}}\right) \\
& \leq(\alpha a+(1-\alpha) b)^{m}-\left(a^{\alpha} b^{1-\alpha}\right)^{m},
\end{aligned}
$$

where $r_{0}=\min \{\alpha, 1-\alpha\}$. This is a considerable new generalization of two refinements of the Young inequality due to Kittaneh and Manasrah, and Hirzallah and Kittaneh, which correspond to the cases $m=1$ and $m=2$, respectively. As applications we give some refined Young type inequalities for generalized euclidean operator radius and the numerical radius of some well-know $f$-connection of operators and refined some Young type inequalities for the traces, determinants, and norms of positive definite matrices.

Mathematics Subject Classification (2010). 26D07, 15A45, 47A30.

Key words and phrases. Young inequality, Positive semidefinite matrix, Euclidean operator radius, $f$ connection.

\section{Introduction}

The arithmetic-geometric mean inequality expressed as follows: For a set of nonnegative real numbers $x_{1}, x_{2}, \ldots, x_{n}$, then

Received : 01 June 2020 - Accepted : 10 July 2020 .

(C) This article is published with open access by Sidi Mohamed Ben Abdallah University.

The Authors: ${ }^{1,2}$ Department of Mathematics, Faculy of Sciences-Semlalia, University Cadi Ayyad, Av. Prince My. Abdellah, BP: 2390, Marrakesh (40.000-Marrakech), Morocco.

e-mail: ${ }^{1}$ mohamedamineighachane@gmail.com

e-mail:2akkm555@yahoo.fr. 


$$
\sqrt[n]{\prod_{k=1}^{n} x_{k}} \leq \sum_{k=1}^{n} \frac{x_{k}}{n}
$$

The weighted form of the arithmetic-geometric mean inequality (AM-GM) states as follows:

Theorem 1.1. Let $n$ be a positive integer. For $k=1,2, \ldots, n$, let $x_{k}>0$, and let $\alpha_{k} \geq 0$ satisfy $\sum_{k=1}^{n} \alpha_{k}=1$. Then, we have

$$
\prod_{k=1}^{n} x_{k}^{\alpha_{k}} \leq \sum_{k=1}^{n} \alpha_{k} x_{k}
$$

The special case of the weighted AM-GM inequality $(n=2)$ is the well-known Young's inequality, for positive real numbers $a, b$ and $0 \leq \alpha \leq 1$, we have

$$
a^{\alpha} b^{1-\alpha} \leq \alpha a+(1-\alpha) b
$$

The first refinements of Young inequality is the squared version proved in [3] as follows

$$
\left(a^{\alpha} b^{1-\alpha}\right)^{2}+r_{0}^{2}(a-b)^{2} \leq(\alpha a+(1-\alpha) b)^{2},
$$

where $r_{0}=\min \{\alpha, 1-\alpha\}$.

Later, Kittaneh and Manasrah [8], obtained the other interesting refinement of Young inequality as follows

$$
a^{\alpha} b^{1-\alpha}+r_{0}(\sqrt{a}-\sqrt{b})^{2} \leq \alpha a+(1-\alpha) b .
$$

S. Furuichi [4] was refined (1.2) as follows:

$$
\prod_{k=1}^{n} x_{k}^{\alpha_{k}}+r\left(\sum_{k=1}^{n} x_{k}-n \sqrt[n]{\prod_{k=1}^{n} x_{k}}\right) \leq \sum_{k=1}^{n} \alpha_{k} x_{k},
$$

where $r=\min \left\{\alpha_{k}: k=1, \ldots, n\right\}$. This inequality generalizes the inequality (1.5).

Recently, Manasrah and Kittaneh [7] gave a generalization of the two refind Young inequalities (1.4) and (1.5) as follows

Theorem 1.2. Let $m$ be a positive integer and let $\alpha$ a positive number such that $0 \leq \alpha \leq 1$. Then we have

$$
\left(a^{\alpha} b^{1-\alpha}\right)^{m}+r_{0}^{m}\left(a^{\frac{m}{2}}-b^{\frac{m}{2}}\right)^{2} \leq(\alpha a+(1-\alpha) b)^{m}
$$

where $r_{0}=\min \{\alpha, 1-\alpha\}$.

This paper is organised as follows: In section 2, by using inequality (1.6) we give a new generalization of the two refined Young inequalities (1.4) and (1.5). In section 3, we apply our main result to establish some inequalities for generalized euclidean operator radius. In section 4 , we show some numerical radius of some well-know $f$-connection of operators. In section 5, we refined some Young type inequalities for the traces, determinants, and norms of positive definite matrices. 


\section{A New generalizations of two refined Young's inequalities}

Before giving the main result, we need the following simple lemma

Lemma 2.1. Let $m$ be a positive integer, and let $\alpha$ a positive number such that $0 \leq \alpha \leq 1$. Then we have

$$
\sum_{k=1}^{m}\left(\begin{array}{c}
m \\
k
\end{array}\right) k \alpha^{k}(1-\alpha)^{m-k}=m \alpha
$$

and

$$
\sum_{k=0}^{m-1}\left(\begin{array}{c}
m \\
k
\end{array}\right)(m-k) \alpha^{k}(1-\alpha)^{m-k}=m(1-\alpha),
$$

where $\left(\begin{array}{c}m \\ k\end{array}\right)$ is the binomial coefficient.

For a proof of Lemma 2.1, one can see [1].

The first main result of this setion is the following theorem.

Theorem 2.1. Let $a$ and $b$ be two positive numbers and $0 \leq \alpha \leq 1$. Then for $m=1,2,3, \ldots$, we have

$$
\left(a^{\alpha} b^{1-\alpha}\right)^{m}+r_{0}^{m}\left(\frac{b^{m+1}-a^{m+1}}{b-a}-(m+1)(a b)^{\frac{m}{2}}\right) \leq(\alpha a+(1-\alpha) b)^{m},
$$

where $r_{0}=\min \{\alpha, 1-\alpha\}$.

Proof. We have,

$$
\begin{aligned}
(\alpha a+(1-\alpha) b)^{m} & =\sum_{k=0}^{m}\left(\begin{array}{c}
m \\
k
\end{array}\right) \alpha^{k}(1-\alpha)^{m-k} a^{k} b^{m-k} \\
& =\sum_{k=0}^{m} \mu_{k} x_{k} .
\end{aligned}
$$

where $x_{k}$ is given by: For $0 \leq k \leq m$,

$$
x_{k}:=a^{k} b^{m-k}, \text { with } \quad \mu_{k}:=\left(\begin{array}{c}
m \\
k
\end{array}\right) \alpha^{k}(1-\alpha)^{m-k} .
$$

We have

(1) $x_{k}>0$ for all $k \in\{0,1, \ldots, m\}$,

(2) $\mu_{k} \geq 0$ for all $k \in\{0,1, \ldots, m\}$, with $\sum_{k=0}^{m} \mu_{k}=1$.

Hence by (1.6),

$$
\begin{aligned}
\sum_{k=0}^{m} \mu_{k} x_{k} & \geq \prod_{k=0}^{m} x_{k}^{\mu_{k}}+r\left(\sum_{k=0}^{m} x_{k}-(m+1)\left(\prod_{k=0}^{m} x_{k}\right)^{\frac{1}{m+1}}\right) \\
& =a^{\alpha(m)} b^{\beta(m)}+r\left(\sum_{k=0}^{m} a^{k} b^{m-k}-(m+1)\left(\prod_{k=0}^{m} a^{k} b^{m-k}\right)^{\frac{1}{m+1}}\right)
\end{aligned}
$$




$$
=a^{\alpha(m)} b^{\beta(m)}+r\left(\frac{b^{m+1}-a^{m+1}}{b-a}-(m+1)(a b)^{\frac{m}{2}}\right)
$$

where

$$
\begin{gathered}
r=\min \left\{\mu_{k}, k=0, \ldots, m\right\}=r_{0}^{m} \\
\alpha(m)=\sum_{k=1}^{m}\left(\begin{array}{c}
m \\
k
\end{array}\right) k \alpha^{k}(1-\alpha)^{m-k}=m \alpha \quad(\text { by Lemma 2.1), }
\end{gathered}
$$

and

$$
\beta(m)=\sum_{k=0}^{m-1}\left(\begin{array}{l}
m \\
k
\end{array}\right)(m-k) \alpha^{k}(1-\alpha)^{m-k}=m(1-\alpha) \quad \text { (by Lemma 2.1). }
$$

Therefore,

$$
\left(a^{\alpha} b^{1-\alpha}\right)^{m}+r_{0}^{m}\left(\frac{b^{m+1}-a^{m+1}}{b-a}-(m+1)(a b)^{\frac{m}{2}}\right) \leq(\alpha a+(1-\alpha) b)^{m} .
$$

Remark 2.1. The Theorem 2.1 is a commun generalization of the inequalities (1.4) and (1.5).

The second main result of this setion is the following theorem.

Theorem 2.2. Let $a$ and $b$ be two positive numbers and $0 \leq \alpha \leq 1$. Then for $m=1,2,3, \ldots$, we have

$$
r_{0}^{m}\left(a^{\frac{m}{2}}-b^{\frac{m}{2}}\right)^{2} \leq r_{0}^{m}\left(\frac{b^{m+1}-a^{m+1}}{b-a}-(m+1)(a b)^{\frac{m}{2}}\right) \leq(\alpha a+(1-\alpha) b)^{m}-\left(a^{\alpha} b^{1-\alpha}\right)^{m},
$$

where $r_{0}=\min \{\alpha, 1-\alpha\}$.

Proof. The second inequality in Theorem 2.2, are special cases of Theorem 2.1. So, it is enough to prove the first inequality. We have,

$$
\begin{gathered}
\left(\frac{b^{m+1}-a^{m+1}}{b-a}-(m+1)(a b)^{\frac{m}{2}}\right)-\left(a^{\frac{m}{2}}-b^{\frac{m}{2}}\right)^{2} \\
=\sum_{k=0}^{m} a^{k} b^{m-k}-(m+1)(a b)^{\frac{m}{2}}-\left(a^{m}+b^{m}-2(a b)^{\frac{m}{2}}\right) \\
=\sum_{k=1}^{m-1} a^{k} b^{m-k}-(m-1)(a b)^{\frac{m}{2}} \\
\geq(m-1)\left(\prod_{k=1}^{m-1} a^{k} b^{m-k}\right)^{\frac{1}{m-1}}-(m-1)(a b)^{\frac{m}{2}} \\
\quad(\text { by inequality }(1.1)) \\
=(m-1)(a b)^{\frac{m}{2}}-(m-1)(a b)^{\frac{m}{2}}=0 .
\end{gathered}
$$


It is known from [9] that for $1 \leq \alpha \leq 1$ and $r \geq 1$,

$$
\alpha a+(1-\alpha) b \leq\left(\alpha a^{r}+(1-\alpha) b^{r}\right)^{1 / r} .
$$

It follows from (2.2) and Theorem 2.1 that

$$
\left(a^{\alpha} b^{1-\alpha}\right)^{m}+r_{0}^{m}\left(\frac{b^{m+1}-a^{m+1}}{b-a}-(m+1)(a b)^{\frac{m}{2}}\right) \leq\left(\alpha a^{r}+(1-\alpha) b^{r}\right)^{\frac{m}{r}},
$$

where $r_{0}=\min \{\alpha, 1-\alpha\}$. In particular, for $\alpha=\frac{1}{2}$, we get

$$
\left(a^{1 / 2} b^{1 / 2}\right)^{m} \leq \frac{1}{2^{\frac{m}{r}}}\left(a^{r}+b^{r}\right)^{\frac{m}{r}}-\frac{1}{2^{m}}\left(\frac{b^{m+1}-a^{m+1}}{b-a}-(m+1)(a b)^{\frac{m}{2}}\right) .
$$

\section{Applications to refined some inequalities for generalized euclidean operator radius}

In this section we provide some improvements to some inequalities due to A. Sheikhhosseini, M Sal Moslehian and K. Shebrawi [13].

Let $B(\mathcal{H})$, denote the $C^{*}$-Algebra of all bounded linear operators acting on a Hilbert space $\mathcal{H}$. If $T \in B(\mathcal{H})$, the numerical radius of $T$ is defined by

$$
w(A)=\sup _{\|x\|=1}|\langle A x, x\rangle| .
$$

The generalized euclidean operator radius $\omega_{p}$ of operators $T_{1}, \ldots, T_{n} \in B(\mathcal{H})$ is defined for $p \geq 1$ as follows (see, [12]):

$$
\omega_{p}\left(T_{1}, \ldots, T_{n}\right):=\sup _{\|x\|=1}\left(\sum_{i=1}^{n}\left|\left\langle T_{i} x, x\right\rangle\right|^{p}\right)^{\frac{1}{p}} .
$$

Before giving our results, we need the following lemmas. The first lemma is known as the mixed Schwarz inequality, this lemma has been proven by Kato [10].

Lemma 3.1. Let $T \in \mathcal{B}(\mathcal{H})$ and $\alpha \in(0,1)$. Then

$$
|\langle T x, y\rangle|^{2} \leq\left\langle|T|^{2 \alpha} x, x\right\rangle\left\langle\left|T^{*}\right|^{2(1-\alpha)} y, y\right\rangle
$$

for all $x, y \in \mathcal{H}$.

The second lemma is a generalization of the mixed Schwarz inequality, this lemma has been proven by F. Kittaneh [6].

Lemma 3.2. Let $T \in \mathcal{B}(\mathcal{H})$ and let $f$ and $g$ be non-negative continuous functions on $[0,+\infty)$ such that $f(t) g(t)=t$ for all $t \in[0,+\infty)$. Then

$$
|\langle T x, y\rangle|^{2} \leq\left\|f(|T|)|||| g\left(\left|T^{*}\right|\right)\right\|,
$$

for all $x, y \in \mathcal{H}$.

The third Lemma follows from spectral theorem for positive operators and Jensen's inequality, this lemma has been proven in [11]. 
Lemma 3.3. (McCarthy inequality) Let $T \in \mathcal{B}(\mathcal{H}) T \geq 0$ and let $x \in \mathcal{H}$ be any unit vector. Then

(a) $\langle T x, x\rangle^{r} \leq\left\langle T^{r} x, x\right\rangle$ for $r \geq 1$

(b) $\left\langle T^{r} x, x\right\rangle \leq\langle T x, x\rangle^{r}$ for $0<r \leq 1$.

The following Theorem is well-known.

Theorem 3.1. [12] Let $A_{i}, B_{i}, T_{i} \in B(\mathcal{H})$ for $i=1,2,3$.., and let $f$ and $g$ be non-negative continuous functions on $[0,+\infty)$ such that $f(t) g(t)=t$, for all $t \in[0,+\infty)$. Then for $r, p \geq 1$, we have

$$
\omega_{p}^{r p}\left(A_{1}^{*} T_{1} B_{1}, . ., A_{n}^{*} T_{n} B_{n}\right) \leq \frac{n^{r-1}}{2}\left\|\sum_{i=1}^{n}\left(\left(\left[B_{i}^{*} f^{2}\left(\left|T_{i}\right|\right) B_{i}\right]^{r p}+\left[A_{i}^{*} g^{2}\left(\left|T_{i}^{*}\right|\right) A_{i}\right]^{r p}\right)\right)\right\| .
$$

The first main result in this section is the following Theorem.

Theorem 3.2. Let $A_{i}, B_{i}, T_{i} \in B(\mathcal{H})$ for $i=1,2,3 .$. , and let $f$ and $g$ be non-negative continuous functions on $[0,+\infty)$ such that $f(t) g(t)=t$, for all $t \in[0,+\infty)$. Then for $m=1,2,3$,.. and $r, p \geq m$, we have

$$
\begin{aligned}
\omega_{p}^{p}\left(A_{1}^{*} T_{1} B_{1}, . ., A_{n}^{*} T_{n} B_{n}\right) & \leq \frac{n^{1-m / r}}{2^{m / r}}\left\|\sum_{i=1}^{n}\left(\left(\left[B_{i}^{*} f^{2}\left(\left|T_{i}\right|\right) B_{i}\right]^{\frac{p r}{m}}+\left[A_{i}^{*} g^{2}\left(\left|T_{i}^{*}\right|\right) A_{i}\right]^{\frac{p r}{m}}\right)\right)\right\|^{\frac{m}{r}} \\
& -\inf _{\|x\|=1} \xi(x),
\end{aligned}
$$

where

$$
\begin{aligned}
\xi(x)=\frac{1}{2^{m}} \sum_{i=1}^{n}\left(\frac{\left\langle\left[B_{i}^{*} f^{2}\left(\left|T_{i}^{*}\right|\right) B_{i}\right]^{\frac{p}{m}} x, x\right\rangle^{m+1}-\left\langle\left[A_{i}^{*} g^{2}\left(\left|T_{i}\right|\right) A_{i}\right]^{\frac{p}{m}} x, x\right\rangle^{m+1}}{\left\langle\left[B_{i}^{*} f^{2}\left(\left|T_{i}^{*}\right|\right) B_{i}\right]^{\frac{p}{m}} x, x\right\rangle-\left\langle\left[A_{i}^{*} g^{2}\left(\left|T_{i}\right|\right) A_{i}\right]^{\frac{p}{m}} x, x\right\rangle}\right. \\
\left.-(m+1)\left[\left\langle\left[A_{i}^{*} g^{2}\left(\left|T_{i}\right|\right) A_{i}\right]^{\frac{p}{m}} x, x\right\rangle\left\langle\left[B_{i}^{*} f^{2}\left(\left|T_{i}^{*}\right|\right) B_{i}\right]^{\frac{p}{m}} x, x\right\rangle\right]^{\frac{m}{2}}\right) .
\end{aligned}
$$

Proof. We have,

$$
\begin{aligned}
\sum_{i=1}^{n}\left|\left\langle A_{i}^{*} T_{i} B_{i} x, x\right\rangle\right|^{p} & =\sum_{i=1}^{n}\left|\left\langle T_{i} B_{i} x, A_{i} x\right\rangle\right|^{p} \\
& \leq\left.\sum_{i=1}^{n}|| f\left(\left|T_{i}\right|\right) B_{i} x||^{p}|| g\left(\left|T_{i}^{*}\right|\right) A_{i} x\right|^{p} \text { (by Lemma 3.2) } \\
& \leq \sum_{i=1}^{n}\left\langle f\left(\left|T_{i}\right|\right) B_{i} x, f\left(\left|T_{i}\right|\right) B_{i} x\right\rangle^{\frac{p}{2}}\left\langle g\left(\left|T_{i}^{*}\right|\right) A_{i} x, g\left(\left|T_{i}^{*}\right|\right) A_{i} x\right\rangle^{\frac{p}{2}} \\
& \leq \sum_{i=1}^{n}\left(\left\langle B_{i}^{*} f^{2}\left(\left|T_{i}\right|\right) B_{i} x, x\right\rangle^{\frac{p}{2 m}}\left\langle A_{i}^{*} g^{2}\left(\left|T_{i}^{*}\right|\right) A_{i} x, x\right\rangle^{\frac{p}{2 m}}\right)^{m} \\
& \leq \sum_{i=1}^{n}\left(\left\langle\left[B_{i}^{*} f^{2}\left(\left|T_{i}\right|\right) B_{i}\right]^{\frac{p}{m}} x, x\right\rangle^{\frac{1}{2}}\left\langle\left[A_{i}^{*} g^{2}\left(\left|T_{i}^{*}\right|\right) A_{i}\right]^{\frac{p}{m}} x, x\right\rangle^{\frac{1}{2}}\right)^{m}
\end{aligned}
$$


(by Lemma 3.3)

$$
\begin{aligned}
& \leq \sum_{i=1}^{n}\left[\frac{1}{2}\left\langle\left[B_{i}^{*} f^{2}\left(\left|T_{i}\right|\right) B_{i}\right]^{\frac{p}{m}} x, x\right\rangle^{r}+\left\langle\left[A_{i}^{*} g^{2}\left(\left|T_{i}^{*}\right|\right) A_{i}\right]^{\frac{p}{m}} x, x\right\rangle^{r}\right]^{\frac{m}{r}}-\xi(x) \\
& \quad \text { by inequality (2.4)) } \\
& \leq \frac{n^{1-m / r}}{2^{m / r}}\left\langle\sum_{i=1}^{n}\left(\left(\left[B_{i}^{*} f^{2}\left(\left|T_{i}^{*}\right|\right) B_{i}\right]^{\frac{p r}{m}}+\left[A_{i}^{*} g^{2}\left(\left|T_{i}^{*}\right|\right) A_{i}\right]^{\frac{p r}{m}}\right)\right) x, x\right\rangle^{\frac{m}{r}}-\xi(x),
\end{aligned}
$$

by lemma 3.3 and the concavity of the function $t \longrightarrow t^{\frac{m}{r}}$. Taking the supremum over $x \in \mathcal{H}$, $\|x\|=1$, we deduce the result. This completes the proof.

Choosing $f(t)=g(t)=\sqrt{t}$, and $T_{i}=I$ for $i=1,2, \ldots, n$, in Theorem 3.2 we obtain the following simpler form.

Corollary 3.1. Let $A_{i}, B_{i}, \in B(\mathcal{H})$ for $(i=1,2,3$..). Then for $m=1,2,3$,.. and $r, p \geq m$, we have

$$
\omega_{p}^{p}\left(A_{1}^{*} B_{1}, . ., A_{n}^{*} B_{n}\right) \leq \frac{n^{1-m / r}}{2^{m / r}} \| \sum_{i=1}^{n}\left(\left(\left|B_{i}\right|^{\frac{2 p r}{m}}+\left|A_{i}\right|^{\frac{2 p r}{m}}\right) \|^{\frac{m}{r}}-\inf _{\|x\|=1} \xi(x)\right.
$$

where

$$
\begin{aligned}
& \xi(x)=\frac{1}{2^{m}} \sum_{i=1}^{n}\left(\frac{\left\langle\left|B_{i}\right|^{\frac{2 p}{m}} x, x\right\rangle^{m+1}-\left\langle\left|A_{i}\right|^{\frac{2 p}{m}} x, x\right\rangle^{m+1}}{\left\langle\left|B_{i}\right|^{\frac{2 p}{m}} x, x\right\rangle-\left\langle\left|A_{i}\right|^{\frac{2 p}{m}} x, x\right\rangle}\right. \\
&\left.-(m+1)\left[\left\langle\left|A_{i}\right|^{\frac{2 p}{m}} x, x\right\rangle\left\langle\left|B_{i}\right|^{\frac{2 p}{m}} x, x\right\rangle\right]^{\frac{m}{2}}\right) .
\end{aligned}
$$

The second result in this section is the following Theorem.

Theorem 3.3. Let $T_{i} \in B(\mathcal{H})$ for $i=1,2,3$.., and $p \geq 2 m$ for some $m=1,2,3, \ldots$ Then for $0 \leq \alpha \leq 1$, we have

$$
\omega_{p}^{p}\left(T_{1}, . ., T_{n}\right) \leq\left\|\sum_{i=1}^{n}\left(\alpha\left|T_{i}\right|^{\frac{p}{m}}+(1-\alpha)\left|T_{i}^{*}\right|^{\frac{p}{m}}\right)^{m}\right\|-\inf _{\|x\|=1} \xi(x),
$$

where

$$
\xi(x)=r_{0}^{m} \sum_{i=1}^{n}\left(\frac{\left\langle\left|T_{i}\right|^{\frac{p}{m}} x, x\right\rangle^{m+1}-\left\langle\left|T_{i}^{*}\right|^{\frac{p}{m}} x, x\right\rangle^{m+1}}{\left\langle\left|T_{i}\right|^{\frac{p}{m}} x, x\right\rangle-\left\langle\left|T_{i}^{*}\right|^{\frac{p}{m}} x, x\right\rangle}-(m+1)\left[\left\langle\left|T_{i}\right|^{\frac{p}{m}} x, x\right\rangle\left\langle\left|T_{i}^{*}\right|^{\frac{p}{m}} x, x\right\rangle\right]^{\frac{m}{2}}\right) .
$$

Proof. We have,

$$
\begin{aligned}
\sum_{i=1}^{n}\left|\left\langle T_{i} x, x\right\rangle\right|^{p} & =\sum_{i=1}^{n}\left(\left|\left\langle T_{i} x, x\right\rangle\right|^{2}\right)^{\frac{p}{2}} \\
& \leq \sum_{i=1}^{n}\left(\left\langle\left|T_{i}\right|^{2 \alpha} x, x\right\rangle\left\langle\left|T_{i}^{*}\right|^{2(1-\alpha)} x, x\right\rangle\right)^{\frac{p}{2}} \text { (by Lemma 3.1) }
\end{aligned}
$$




$$
\begin{aligned}
& \leq \sum_{i=1}^{n}\left(\left\langle\left|T_{i}\right|^{\frac{p \alpha}{m}} x, x\right\rangle\left\langle\left|T_{i}^{*}\right|^{\frac{p(1-\alpha)}{m}} x, x\right\rangle\right)^{m} \text { (by Lemma 3.3) } \\
& \leq \sum_{i=1}^{n}\left(\left\langle\left|T_{i}\right|^{\frac{p}{m}} x, x\right\rangle^{\alpha}\left\langle\left|T_{i}^{*}\right|^{\frac{p}{m}} x, x\right\rangle^{(1-\alpha)}\right)^{m}(\text { by Lemma 3.2) } \\
& \leq \sum_{i=1}^{n}\left(\alpha\left\langle\left|T_{i}\right|^{\frac{p}{m}} x, x\right\rangle+(1-\alpha)\left\langle\left|T_{i}^{*}\right|^{\frac{p}{m}} x, x\right\rangle\right)^{m}-\zeta(x)
\end{aligned}
$$

(by Theorem 2.1)

$$
\begin{aligned}
& \leq \sum_{i=1}^{n}\left(\left\langle\left(\alpha\left|T_{i}\right|^{\frac{p}{m}}+(1-\alpha)\left|T_{i}^{*}\right|^{\frac{p}{m}}\right) x, x\right\rangle\right)^{m}-\inf _{\|x\|=1} \zeta(x) \\
& \leq \sum_{i=1}^{n}\left\langle\left(\alpha\left|T_{i}\right|^{\frac{p}{m}}+(1-\alpha)\left|T_{i}^{*}\right|^{\frac{p}{m}}\right)^{m} x, x\right\rangle-\inf _{\|x\|=1} \zeta(x)
\end{aligned}
$$

(by Lemma 3.3)

$$
\leq\left\langle\sum_{i=1}^{n}\left(\alpha\left|T_{i}\right|^{\frac{p}{m}}+(1-\alpha)\left|T_{i}^{*}\right|^{\frac{p}{m}}\right)^{m} x, x\right\rangle-\inf _{\|x\|=1} \zeta(x) \text {. }
$$

Taking the supremum over $x \in \mathcal{H},\|x\|=1$, we deduce the result. This completes the proof.

\section{Some results for $f$-connections of operators}

For positive definite operators $A, B \in B(\mathcal{H})$, the operator geometric mean is defined by

$$
A \sharp B=A^{1 / 2}\left(A^{-1 / 2} B A^{-1 / 2}\right)^{1 / 2} A^{1 / 2} \text {. }
$$

Lef $f$ be a continuous function defined on the real interval $J$ containing the spectrum of the operator $A^{-1 / 2} B A^{-1 / 2}$, where $B$ is a self-adjoint operators and $A$ is a positive intertible operator. By using the continuous functional calculus, Tafazoli et al. [14] defined $f$-connection $\sigma_{f}$ as follows:

$$
A \sigma_{f} B=A^{1 / 2} f\left(A^{-1 / 2} B A^{-1 / 2}\right) A^{1 / 2} .
$$

The aim of this section is to give some refinements of numerical radius inequalities for the $f$-connection of operators [14].

The first main result of this section which can be reads as follows.

Theorem 4.1. Let $A, B, X \in B(\mathcal{H})$ be such that $A, B$ are positive definite. Then for all integer $m$, and $r \geq 1$, we have

$$
w^{m}\left(\left(A \sigma_{f} B\right) X\right) \leq 2^{\frac{-m}{r}} w^{\frac{m}{r}}\left(\left(X^{*} A^{1 / 2} f^{2}\left(A^{-1 / 2} B A^{-1 / 2}\right) A^{1 / 2} X\right)^{r}+A^{r}\right)-\inf _{\|x\|=1} \xi(x),
$$

where

$$
\xi(x)=\frac{1}{2^{m}}\left(\frac{\left\langle X^{*} A^{1 / 2} f^{2}\left(A^{-1 / 2} B A^{-1 / 2}\right) A^{1 / 2} X x, x\right\rangle^{m+1}-\langle A x, x\rangle^{m+1}}{\left\langle X^{*} A^{1 / 2} f^{2}\left(A^{-1 / 2} B A^{-1 / 2}\right) A^{1 / 2} X x, x\right\rangle-\langle A x, x\rangle}\right.
$$




$$
\left.-(m+1)\left(\left\langle X^{*} A^{1 / 2} f^{2}\left(A^{-1 / 2} B A^{-1 / 2}\right) A^{1 / 2} X x, x\right\rangle\langle A x, x\rangle\right)^{\frac{m}{2}}\right) .
$$

Proof. We have,

$$
\begin{aligned}
\left.\left|\left\langle A \sigma_{f} B\right) X x, x\right\rangle\right|^{m}= & \left|\left\langle A^{1 / 2} f\left(A^{-1 / 2} B A^{-1 / 2}\right) A^{1 / 2} X x, x\right\rangle\right|^{m} \\
\leq & \left\|f\left(A^{-1 / 2} B A^{-1 / 2}\right) A^{1 / 2} X x\right\|^{m}\left\|A^{1 / 2} x\right\|^{m} \\
\leq & \left(\left\langle f\left(A^{-1 / 2} B A^{-1 / 2}\right) A^{1 / 2} X x, f\left(A^{-1 / 2} B A^{-1 / 2}\right) A^{1 / 2} X x\right\rangle^{\frac{1}{2}}\left\langle A^{1 / 2} x, A^{1 / 2} x\right\rangle^{\frac{1}{2}}\right)^{m} \\
\leq & \leq\left(\left\langle X^{*} A^{1 / 2} f^{2}\left(A^{-1 / 2} B A^{-1 / 2}\right) A^{1 / 2} X x, x\right\rangle^{\frac{1}{2}}\langle A x, x\rangle^{\frac{1}{2}}\right)^{m} \\
\leq & \leq 2^{\frac{-m}{r}}\left(\left\langle X^{*} A^{1 / 2} f^{2}\left(A^{-1 / 2} B A^{-1 / 2}\right) A^{1 / 2} X x, x\right\rangle^{r}+\langle A x, x\rangle^{r}\right)^{\frac{m}{r}}-\xi(x) \\
& (\text { by inequality }(2.4)) \\
\leq & 2^{\frac{-m}{r}}\left(\left\langle\left(\left(X^{*} A^{1 / 2} f^{2}\left(A^{-1 / 2} B A^{-1 / 2}\right) A^{1 / 2} X\right)^{r}+A^{r}\right) x, x\right\rangle\right)^{\frac{m}{r}}-\xi(x) \\
& \quad(\text { by Lemma 3.3). }
\end{aligned}
$$

Taking the supremum over $x \in \mathcal{H},\|x\|=1$, we deduce the result.

Taking $X=I$, in Theorem 4.1, then we have the following corollary

Corollary 4.1. Let $A, B \in B(\mathcal{H})$ be such that $A, B$ are positive definite. Then for all integers $m$, and $r \geq 1$, we have

$$
\left.w\left(A \sigma_{f} B\right) \leq 2^{\frac{-m}{r}} w^{\frac{m}{r}}\left(A^{1 / 2} f^{2}\left(A^{-1 / 2} B A^{-1 / 2}\right) A^{1 / 2}\right)^{r}+A^{r}\right)-\inf _{\|x\|=1} \xi(x),
$$

where

$$
\begin{gathered}
\xi(x)=\frac{1}{2^{m}}\left(\frac{\left\langle A^{1 / 2} f^{2}\left(A^{-1 / 2} B A^{-1 / 2}\right) A^{1 / 2} x, x\right\rangle^{m+1}-\langle A x, x\rangle^{m+1}}{\left\langle A^{1 / 2} f^{2}\left(A^{-1 / 2} B A^{-1 / 2}\right) A^{1 / 2} x, x\right\rangle-\langle A x, x\rangle}\right. \\
\left.-(m+1)\left(\left\langle A^{1 / 2} f^{2}\left(A^{-1 / 2} B A^{-1 / 2}\right) A^{1 / 2} x, x\right\rangle\langle A x, x\rangle\right)^{\frac{m}{2}}\right) .
\end{gathered}
$$

Taking $r=1, X=I$, and $f(t)=\sqrt{t}$, in Theorem 4.1, we have the following simplified form. Corollary 4.2. Let $A, B \in B(\mathcal{H})$ be such that $A, B$ are positive definite. Then for all integers $m$, we have

$$
w^{m}(A \sharp B) \leq 2^{-m} w^{m}(A+B)-\inf _{\|x\|=1} \xi(x),
$$

where

$$
\xi(x)=\frac{1}{2^{m}}\left(\frac{\langle B x, x\rangle^{m+1}-\langle A x, x\rangle^{m+1}}{\langle B x, x\rangle-\langle A x, x\rangle}-(m+1)(\langle A x, x\rangle\langle B x, x\rangle)^{\frac{m}{2}}\right) .
$$

The second main result of this section which can be reads as follows. 
Theorem 4.2. Let $A, B, X \in B(\mathcal{H})$ be such that $A, B$ are positive definite. Then for all integers $m$, and $r>1$, we have

$$
\left\|\left(A \sigma_{f} B\right) X\right\|^{m} \leq 2^{\frac{-m}{r}}\left(\|\left(X^{*} A^{1 / 2} f^{2}\left(A^{-1 / 2} B A^{-1 / 2}\right) A^{1 / 2} X\left\|^{r}+\right\| A \|^{r}\right)^{\frac{m}{r}}-\inf _{\|x\|=1,\|y\|=1} \xi(x, y),\right.
$$

where

$$
\begin{aligned}
\xi(x, y)= & \frac{1}{2^{m}}\left(\frac{\left\langle X^{*} A^{1 / 2} f^{2}\left(A^{-1 / 2} B A^{-1 / 2}\right) A^{1 / 2} X x, x\right\rangle^{m+1}-\langle A y, y\rangle^{m+1}}{\left\langle X^{*} A^{1 / 2} f^{2}\left(A^{-1 / 2} B A^{-1 / 2}\right) A^{1 / 2} X x, x\right\rangle-\langle A y, y\rangle}\right. \\
& \left.-(m+1)\left(\left\langle X^{*} A^{1 / 2} f^{2}\left(A^{-1 / 2} B A^{-1 / 2}\right) A^{1 / 2} X x, x\right\rangle\langle A y, y\rangle\right)^{\frac{m}{2}}\right) .
\end{aligned}
$$

Proof. We have,

$$
\begin{aligned}
\left.\left|\left\langle A \sigma_{f} B\right) X x, y\right\rangle\right|^{m} & =\left|\left\langle A^{1 / 2} f\left(A^{-1 / 2} B A^{-1 / 2}\right) A^{1 / 2} X x, y\right\rangle\right|^{m} \\
& \leq\left|\left\langle f\left(A^{-1 / 2} B A^{-1 / 2}\right) A^{1 / 2} X x, A^{1 / 2} y\right\rangle\right|^{m} \\
& \leq\left(\left\langle f\left(A^{-1 / 2} B A^{-1 / 2}\right) A^{1 / 2} X x, f\left(A^{-1 / 2} B A^{-1 / 2}\right) A^{1 / 2} X x\right\rangle^{\frac{1}{2}}\left\langle A^{1 / 2} y, A^{1 / 2} y\right\rangle^{\frac{1}{2}}\right)^{m} \\
& \leq\left(\left\langle X^{*} A^{1 / 2} f^{2}\left(A^{-1 / 2} B A^{-1 / 2}\right) A^{1 / 2} X x, x\right\rangle^{\frac{1}{2}}\langle A y, y\rangle^{\frac{1}{2}}\right)^{m} \\
& \leq 2^{\frac{-m}{r}}\left(\left\langle X^{*} A^{1 / 2} f^{2}\left(A^{-1 / 2} B A^{-1 / 2}\right) A^{1 / 2} X x, x\right\rangle^{r}+\langle A y, y\rangle^{r}\right)^{\frac{m}{r}}-\xi(x, y)
\end{aligned}
$$

(by inequality (2.4)).

Taking the supremum over $x, y \in \mathcal{H},\|x\|=\|y\|=1$, we deduce the result.

Taking $f(t)=\sqrt{t}$, in Theorem 4.2, we have the following simplified form.

Corollary 4.3. Let $A, B, X \in B(\mathcal{H})$ be such that $A, B$ are positive definite. Then for all integers $m$, and $r>1$, we have

$$
\|(A \sharp B) X\|^{m} \leq 2^{-m}\left(\left\|X^{*} B X\right\|^{r}+\|A\|^{r}\right)^{\frac{m}{r}}-\inf _{\|x\|=1,\|y\|=1} \xi(x, y),
$$

where

$$
\xi(x, y)=\frac{1}{2^{m}}\left(\frac{\left\langle X^{*} B X x, x\right\rangle^{m+1}-\langle A y, y\rangle^{m+1}}{\left\langle X^{*} B X x, x\right\rangle-\langle A y, y\rangle}-(m+1)\left(\langle A y, y\rangle\left\langle X^{*} B X x, x\right\rangle\right)^{\frac{m}{2}}\right) .
$$

\section{Applications to refined Young type inequalities for traces, determinants, and norms of positive definite matrices}

In this section, we give some refined Young type inequalities for traces, determinants, and norms of positive definite matrices.

Let $\mathbf{M}_{n}(\mathbb{C})$ be the space of $n \times n$ complex matrices. A matrix $A \in \mathbf{M}_{n}(\mathbb{C})$ is called positive semidefinite, denoted as $A \geq 0$ if $x^{*} A x \geq 0$ for all $x \in \mathbb{C}^{n}$, and it is called positive definite denoted as $A>0$ if $x^{*} A x>0$ for all nonzero $x \in \mathbb{C}^{n}$. The singular values of a matrix 
$A \in \mathbf{M}_{n}(\mathbb{C})$ are the eigenvalues of the positive semidefinite matrix $|A|=\left(A^{*} A\right)^{1 / 2}$, denoted by $s_{i}(A)$ for $i=1,2,3 . ., n$. A norm \|\|$. \|$, on $\mathbf{M}_{n}(\mathbb{C})$ is called unitarily invariant if $\|U A V\|=$ $\|A\|$ for all $A \in \mathbf{M}_{n}(\mathbb{C})$ and all unitary matrices $U, V \in \mathbf{M}_{n}(\mathbb{C})$.

The trace norme $\|$.$\| is given by \|A\|_{1}=t r|A|=\sum_{k=1}^{n} s_{k}(A)$, where $t r$ is the usual trace. This norm is unitarily invariant.

A matrix Young's inequality due to Ando [2] asserts that

$$
s_{j}\left(A^{\alpha} B^{1-\alpha}\right) \leq s_{j}(\alpha A+(1-\alpha) B),
$$

the above singular value inequality entails the following unitarily invarant norm inequality

$$
\left\|A^{\alpha} B^{1-\alpha}\right\|\|\leq\| \mid \alpha A+(1-\alpha) B\|\| .
$$

A determinant version of Young's inequalities is also known [11; p. 467]: For positive semidefinite matrices $A, B$ and $0 \leq \alpha \leq 1$,

$$
\operatorname{det}\left(A^{\alpha} B^{1-\alpha}\right) \leq \operatorname{det}(\alpha A+(1-\alpha) B) \text {. }
$$

To prove the result of this section, we need the following two lemmas, the first lemma (see, e.g., [11; p. 482,]) is the Minkowski inequality for determinants. the second lemma [5] is a Heinz-Kato type inequality for unitarily invariant norms.

Lemma 5.1. Let $A, B \in \mathbf{M}_{n}(\mathbb{C})$ be positive definite matrices. Then we have

$$
\operatorname{det}(A+B)^{\frac{1}{n}} \geq \operatorname{det}(A)^{\frac{1}{n}}+\operatorname{det}(B)^{\frac{1}{n}} .
$$

Lemma 5.2. Let $A, B \in \mathbf{M}_{n}(\mathbb{C})$ be positive semidefinite matrices. Then we have

$$
\left\|A^{\alpha} X B^{1-\alpha}\right\| \mid \leq\|A X\|^{\alpha}\|\| X B \|^{1-\alpha} .
$$

In particuler

$$
\operatorname{tr}\left|A^{\alpha} X B^{1-\alpha}\right| \leq(\operatorname{tr} A)^{\alpha}(\operatorname{tr} B)^{1-\alpha}
$$

The first result of this section concerns the determinat of positive definite matrices which can be reads as follows:

Theorem 5.1. Let $A, B \in \mathbf{M}_{n}(\mathbb{C})$ be positive definite matrices and $0 \leq \alpha \leq 1$. Then for $m=$ $1,2,3, \ldots$, we have

$$
\begin{aligned}
\left(\operatorname{det}\left(A^{\alpha} B^{1-\alpha}\right)\right)^{m} & +r_{0}^{n m}\left(\frac{\operatorname{det}(B)^{m+\frac{1}{n}}-\operatorname{det}(A)^{m+\frac{1}{n}}}{\operatorname{det}(B)^{\frac{1}{n}}-\operatorname{det}(A)^{\frac{1}{n}}}-(m+1)(\operatorname{det}(A B))^{\frac{m}{2}}\right) \\
& \leq \operatorname{det}(\alpha A+(1-\alpha) B)^{m}
\end{aligned}
$$

where $r_{0}=\min \{\alpha, 1-\alpha\}$.

Proof. We have

$$
\begin{aligned}
\operatorname{det}(\alpha A+(1-\alpha) B)^{m} & =\left[\operatorname{det}(\alpha A+(1-\alpha) B)^{\frac{1}{n}}\right]^{n m} \\
& \geq\left[\operatorname{det}(\alpha A)^{\frac{1}{n}}+\operatorname{det}((1-\alpha) B)^{\frac{1}{n}}\right]^{n m}(\text { by Lemma 5.1) }
\end{aligned}
$$




$$
\begin{aligned}
& =\left[\alpha \operatorname{det}(A)^{\frac{1}{n}}+(1-\alpha) \operatorname{det}(B)^{\frac{1}{n}}\right]^{n m} \\
& \geq\left[\left(\operatorname{det}(A)^{\frac{1}{n}}\right)^{\alpha}\left(\operatorname{det}(B)^{\frac{1}{n}}\right)^{1-\alpha}\right]^{n m} \\
& +r_{0}^{n m}\left(\frac{\operatorname{det}(B)^{m+\frac{1}{n}}-\operatorname{det}(A)^{m+\frac{1}{n}}}{\operatorname{det}(B)^{\frac{1}{n}}-\operatorname{det}(A)^{\frac{1}{n}}}-(m+1)(\operatorname{det}(A B))^{\frac{m}{2}}\right)
\end{aligned}
$$

(by Theorem 2.1)

$$
\begin{aligned}
& =\left(\operatorname{det}\left(A^{\alpha} B^{1-\alpha}\right)\right)^{m} \\
& +r_{0}^{n m}\left(\frac{\operatorname{det}(B)^{m+\frac{1}{n}}-\operatorname{det}(A)^{m+\frac{1}{n}}}{\operatorname{det}(B)^{\frac{1}{n}}-\operatorname{det}(A)^{\frac{1}{n}}}-(m+1)(\operatorname{det}(A B))^{\frac{m}{2}}\right) .
\end{aligned}
$$

The second result of this section concerns the traces of positive definite matrices which can be reads as follows:

Theorem 5.2. Let $A, B \in \mathbf{M}_{n}(\mathbb{C})$ be positive definite matrices and $0 \leq \alpha \leq 1$. Then for $m=$ $1,2,3, \ldots$, we have

$$
\begin{aligned}
\left(\operatorname{tr}\left(\left|A^{\alpha} B^{1-\alpha}\right|\right)^{m}\right. & +r_{0}^{m}\left(\frac{(\operatorname{tr} B)^{m+1}-(\operatorname{tr} A)^{m+1}}{(\operatorname{tr} B)-(\operatorname{tr} A)}-(m+1)((\operatorname{tr} A)(\operatorname{tr} B))^{\frac{m}{2}}\right) \\
& \leq[\operatorname{tr}(\alpha A+(1-\alpha) B)]^{m},
\end{aligned}
$$

where $r_{0}=\min \{\alpha, 1-\alpha\}$.

Proof. We have

$$
\begin{aligned}
\left(\operatorname{tr}\left(\left|A^{\alpha} B^{1-\alpha}\right|\right)^{m}\right. & +r_{0}^{m}\left(\frac{(\operatorname{tr} B)^{m+1}-(\operatorname{tr} A)^{m+1}}{(\operatorname{tr} B)-(\operatorname{tr} A)}-(m+1)((\operatorname{tr} A)(\operatorname{tr} B))^{\frac{m}{2}}\right) \\
\leq & {\left[(\operatorname{tr} A)^{\alpha}(\operatorname{tr} B)^{1-\alpha}\right]^{m}+r_{0}^{m}\left(\frac{(\operatorname{tr} B)^{m+1}-(\operatorname{tr} A)^{m+1}}{(\operatorname{tr} B)-(\operatorname{tr} A)}-(m+1)((\operatorname{tr} A)(\operatorname{tr} B))^{\frac{m}{2}}\right) }
\end{aligned}
$$

(by inequality (5.4))

$$
\leq[\operatorname{tr}(\alpha A+(1-\alpha) B)]^{m} \text { (by Theorem 2.1). }
$$

The third result of this section concerns the norms of positive semi-definite matrices which can be reads as follows:

Theorem 5.3. Let $A, X, B \in \mathbf{M}_{n}(\mathbb{C})$ be positive semidefinite matrices and $0 \leq \alpha \leq 1$. Then for $m=1,2,3, \ldots$.

$$
\|\left.\left|A^{\alpha} X B^{1-\alpha}\right|\right|^{m}+r_{0}^{m}\left(\frac{(\| X B|| \mid)^{m+1}-(|||A X|||)^{m+1}}{(|||X B|||)-(|||A X|||)}-(m+1)((\||| A X|| \mid)(|||X B|||))^{\frac{m}{2}}\right)
$$




$$
\leq[\alpha\|\| A X\|+(1-\alpha)\| X B \|]^{m},
$$

where $r_{0}=\min \{\alpha, 1-\alpha\}$.

Proof. We have

$$
\begin{aligned}
& \|\left|A^{\alpha} X B^{1-\alpha}\right|||^{m}+r_{0}^{m}\left(\frac{(|| X B|| \mid)^{m+1}-(|||A X|||)^{m+1}}{(|||X B|||)-(|||A X|||)}-(m+1)((|||A X|||)(|||X B|||))^{\frac{m}{2}}\right) \\
& \leq\left[\|\| A X\|\|^{\alpha}\|X B\|^{1-\alpha}\right]^{m} \\
& +r_{0}^{m}\left(\frac{(\| X B|| \mid)^{m+1}-(|||A X|||)^{m+1}}{(|||X B|||)-(|||A X|||)}-(m+1)((|||A X|||)(|||X B|||))^{\frac{m}{2}}\right)
\end{aligned}
$$

( by inequality (5.2))

$$
\leq\left[\alpha \| \| A X \left\||+(1-\alpha)\||| X B\|]^{m}(\text { by Theorem } 2.1)\right.\right. \text {. }
$$

Acknowledgements. The authors would like to express their deep thanks to the anonymous referees for their comments and suggestions on the initial version of the manuscript which lead to the improvement of this paper.

\section{References}

[1] M. Akkouchi and M. A. Ighachane, A new proof of a refined Young inequality, Bull. Int. Math. Virtual Inst,. Vol. 10(3) (2020), 425-428.

[2] T. Ando, Matrix Young inequality, per. Theory Adv. Appl. 75,33-38(1995)

[3] O. Hirzallah, and F. Kittaneh, Matrix Young inequalities for the Hilbert-Schmidt norm, Linear Algebra Appl. 308(2000) 77-84.

[4] S. Furuichi, On refined Young inequalities and reverse inequalities, J. Math. Inequal. 5(2011) 21-30.

[5] F. Kittaneh, Norm inequalities for fractionl powers of posetive operators, Lett. Math. Phys. 27,279-285

[6] F. Kittaneh, Notes on some inequalities for Hilbert space operators, Publ, Res. Inst, Math, Sci, 24(1998), 283-203.

[7] F. Kittaneh, and Y. Manasrah, A generalization of two refined Young inequalities, Positivity 19(2015) 757-768.

[8] F. Kittaneh, and Y. Manasrah, Improved Young and Heinz inequalities for matrices, J. Math. Anal. Appl. 36(2010) 292-269.

[9] G. H. Hardy, J. E. Littlewood, and G. Polya, Inequalities, 2nd ed., Cambridge Univ. Press, Cambridge, 1988.

[10] T. Kato Notes on some inequalities for linear operators,Math Ann. 1952; 125:208-212.

[11] C. A. McCarthy, $c_{p}$, Israel, J. Math. 5 (1967), 249-271. Horn, R. A., Johnson, C.R, Matrix analysis, Cambridge Univ. Press, New-York, (1985).

[12] M. Sattari, M.S. Moslehian, K. Shebrawi, Extension of Euclidean operator radius inequalities, Math. Scand. (2016), in press, arXive: 1502.00083 .

[13] A. Sheikhhosseini, K. Shebrawi and M.S. Moslehian, inequalities for generalized Euclidean operator radius via Young's inequality , J. Math. Anal. Appl 445 (2017) 1516-1529.

[14] S. Tafazoli, HR. Moradi, and S. Furuichi, Further inequalities for the numerical radius oh hilbert space operators, J. Math. Ineq. 13(2019). DOI:a0.7153/jmi-2019-13-68. 